\title{
Effects of Skin Surface Temperature on Photoplethysmograph
}

\author{
In Cheol Jeong', Hyungro Yoon ${ }^{2}$, Hyunjeong Kang ${ }^{3}$ and Hojun Yeom ${ }^{4 *}$ \\ ${ }^{1}$ Division of Geriatric Medicine and Gerontology, Johns Hopkins University School of \\ Medicine, Baltimore, MD, USA \\ ${ }^{2}$ Department of Biomedical Engineering, Yonsei University, Wonju, Kangwon, \\ South Korea \\ ${ }^{3}$ Department of Biological Sciences, Korea Advanced Institute of Science and \\ Technology, Daejeon, South Korea \\ ${ }^{4}$ Department of Medical Engineering, Eulji University, Seongnam,Gyeonggi, \\ South Korea
}

Submitted February 2014. Accepted for publication September 2014.

\begin{abstract}
Photoplethysmograph (PPG) has been widely used to investigate various cardiovascular conditions. Previous studies demonstrated effects of temperature of the measurement environment; however, an integrated evaluation has not been established in environments with gradual air temperature variation. The purpose of this study is to investigate variations and relationships of blood pressure (BP), PPG and cardiovascular parameters such as heart rate (HR), stroke volume (SV), cardiac output (CO) and total peripheral resistance (TPR), by changing skin surface temperature (SST). Local mild cooling and heating was conducted on 16 healthy subjects. The results showed that local SST changes affected Finometer blood pressures (Finger BP), PPG components and TPR, but not the oscillometric blood pressure (Central BP), HR, SV and CO, and indicated that temperature must be maintained and monitored to reliably evaluate cardiovascular conditions in temperature-varying environments.
\end{abstract}

Keywords: Photoplethysmography, local cooling and heating, skin surface temperature, cardiovascular status, vasoconstriction, vasodilation

\section{INTRODUCTION}

Photopletysmograph (PPG) is an optical technique that can be used to non-invasively observe changes in blood flow in peripheral blood vessels. PPG indirectly measures cardiovascular conditions through aspects such as scattering, absorption, reflection, transmission, and fluorescence using the optical sensor with a light source [1].

*Corresponding author: Hojun Yeom, 212 Yanggidong, Sujeonggu, Sungnamsi, Gyounggido, South Korea. Phone: (031) 740-7462.E-mail: hyeom@eulji.ac.kr. Other authors: ijeong1@jhmi.edu; hryoon@yonsei .ac.kr; hjkang12@kaist.ac.kr. 
PPG is classified into the alternating current (AC) component and the direct current (DC) component. The AC component generally reflects changes in blood flow at each heart beat and the DC component reflects activities of autonomic nerve system. Previous studies have demonstrated that PPG in peripheral region is similar to central pressure pulses in the aortic root and the ascending aorta [2-5], and PPG can provide various estimated cardiovascular conditions [6-7] even if the cause of changes in PPG has not been fully found. PPG components have been used to detect diseases of the peripheral blood vessels which affect arterial blood circulation, assess blood pressure (BP), cardiac output (CO) and the elasticity of blood vessels, and measure arterial oxygen saturation [1].

With the development of computer-based digital signal processing, PPG technique is being used in diverse areas, especially in areas requiring mobile technology. However, PPG is affected by external factors such as method of attaching probes on skin, pressure between a probe and the contact area, subject's alertness and environment adaptation, and temperature, because of the optical interaction between the light source and the measurement area [1]. Therefore, PPG should be considered by external factors to evaluate more reliable cardiovascular conditions [4, 8-9].

The purpose of this pilot study is to investigate variations and relationships of BP, PPG and cardiovascular parameters such as heart rate (HR), stroke volume (SV), CO and total peripheral resistance (TPR), by changing skin surface temperature (SST) through local gradual cooling and heating. To compare diverse changes in PPG and cardiovascular parameters that relate to changes in SST of measurement area, the air temperature in the chamber was controlled. Evaluation was made by statistical comparisons among temperatures, PPG components and cardiovascular parameters.

\section{METHODS}

\subsection{Systems}

\subsubsection{Cooling and Heating Chamber}

A polypropylene chamber was designed to control inside temperature and to isolate outside air temperature. A hand hole was covered by silicon, and transparent acrylic observation windows were provided toward the participant and on the opposite side to confirm the positioning of left hand. A small fan was installed at the entrance of each of the four cylinders in the chamber to smoothly send warm or cold air that came from the central air conditioning device. Eleven small holes were provided at the base of each cylinder to evenly distribute heating or cooling air and to prevent direct exposures. Three small air holes were provided on the opposite of heating or cooling air inlet (Figure 1(a)).

\subsubsection{PPG Sensor}

Clip type PPG probes (DS-100A, Nellcor, Japan) were used to minimize the effects on the contact pressure and the distance between the light-emitting diode (LED) and the photo-detector; the probes only measure changes in PPG components by inducing changes in SST. PPG were detected on both index fingers (Figure 1(c)). Analog $20 \mathrm{~Hz}$ low-pass filter and $0.15 \mathrm{~Hz}$ high-pass filter were applied to extract AC and DC 
(a)

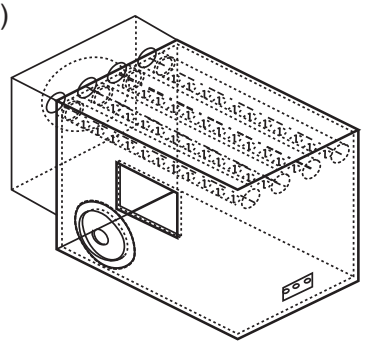

(b)

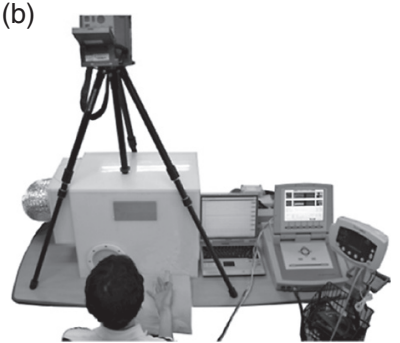

(c)

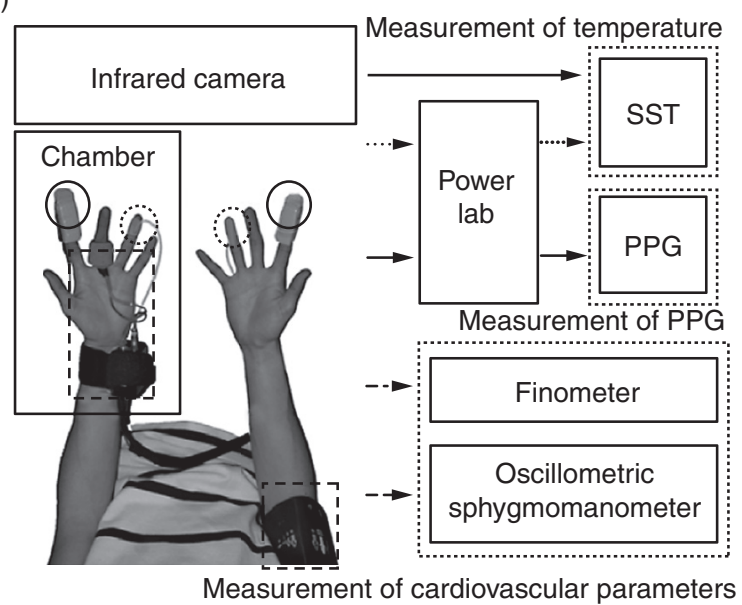

Figure 1. Experimental facilities. (a) Cooling and heating chamber. (b) Experimental environment. (c) Sensors and positions.

components. Data was sampled at $200 \mathrm{~Hz}$ with Power Lab (ML880, AD Instrumentation, Australia). The current supplied to LED drive and the amplification ratio were both held constant.

\subsubsection{Cardiovascular Parameter Sensors}

Finometer (Model-1, FMS, Netherlands) was used to continuously measure BP, HR, SV, CO and TPR while changing SST [10-14]. Sensors of Finometer were positioned on the left middle finger. BP on the right upper arm was periodically measured by Oscillometric sphygmomanometer (Model-53000, Welchallyn, USA) (Figure 1(b), (c)).

\subsubsection{Temperature Sensors}

Two contact temperature sensors (LNTG473FW, Lattron, Korea) were used to measure SST due to changes in chamber air temperature and positioned on both fourth fingers. Power Lab sampled temperature data at $200 \mathrm{~Hz}$. An infrared camera (TH5104, NEC, Japan) was used to measure SST distributions of both hands (Figure 1(c)). 


\subsection{Experiment}

\subsubsection{Subjects}

Sixteen healthy participants (Table 1) were enrolled this experiment. All participants were not undergoing any drug treatment or experiencing Raynaud`s phenomenon. Before the experiment, all participants received a training program and signed a research informed consent. The experimental protocol was reviewed and approved by the Institutional Review Board, Wonju, Yonsei University.

All participants strictly refrained from smoking, alcohol consumption and extreme exercise for $12 \mathrm{~h}$, and refrained from caffeine intake for $4 \mathrm{~h}$ before the experiment to minimize unexpected effects which could affect blood flow and blood vessel conditions, in order to obtain more reliable data due to changes in SST [15].

\subsubsection{Procedure}

After sensors installation, participants were seated in a comfortable chair and both hands were placed on the table at the same level of heart. The left hand was totally inside the chamber and the right hand was positioned outside of the chamber. For the first $10 \mathrm{~min}$ of procedure, data were obtained in an environment at the same temperature as the laboratory, and then data were obtained for another 10 min while the chamber air temperature was changed. During the procedure, the chamber air temperature and participant's PPG components, Finometer BP, HR, SV, CO, TPR and SST were continuously measured, and participant's oscillometric BP at 0, 2.5, 7.5, 12.5 and $17.5 \mathrm{~min}$ and SST distribution at $0,5,10,15$ and $20 \mathrm{~min}$ were periodically measured. Every participant was tested under both heating and cooling conditions.

\subsection{Analyses}

Statistical analyses were conducted to evaluate the changes in PPG components and cardiovascular parameters due to changes in the local SST. Means and standard deviations of PPG components and cardiovascular parameters were calculated to investigate variations in both the time domain and the temperature domain. Pairedsamples tests were performed to compare before and after data changes. Correlations between SST and PPG components and between SST and cardiovascular parameters

Table 1. Demographic and physical characteristics of $\operatorname{subjects}(n=16)$

\begin{tabular}{lccc}
\hline Variables & Min & Max & Mean \pm SD \\
\hline Age (years) & 23 & 30 & $26 \pm 2.1$ \\
Height $(\mathrm{cm})$ & 167 & 184 & $174 \pm 4.1$ \\
Weight $(\mathrm{kg})$ & 60 & 85 & $72 \pm 6.5$ \\
BMI $\left(\mathrm{kg} / \mathrm{m}^{2}\right)$ & 20.1 & 26 & $23 \pm 4.8$ \\
Resting HR (beats/min) & 57.7 & 82.5 & $73 \pm 8.8$ \\
\hline
\end{tabular}

BMI: body mass index, HR: heart rate. 
were analyzed to find influences of SST. SPSS 12.0 for Window was employed for all the analyses.

\section{RESULTS}

\subsection{BPs and Temperatures}

During the cooling period, the chamber air temperature was decreased to $-9.4 \pm 0.7^{\circ} \mathrm{C}, \mathrm{p}$ $<0.01)$. With the chamber temperature decrease, the left SST $\left(-8.8 \pm 2.5^{\circ} \mathrm{C}, \mathrm{p}<0.01\right)$ and the right SST $(-1.6 \pm 1.4, \mathrm{p}<0.01)$ were significantly decreased. Both Finometer systolic blood pressure $(\mathrm{SBP})(10 \pm 9.0 \mathrm{mmHg}, \mathrm{p}<0.01)$ and diastolic blood pressure (DBP) $(7 \pm 6.0 \mathrm{mmHg}, \mathrm{p}<0.01)$ significantly increased. However, oscillometric SBP and DBP did not show significant changes.

During the heating period, the chamber air temperature was increased to $19.5 \pm 1.2^{\circ} \mathrm{C}$ $(\mathrm{p}<0.01)$. With the chamber temperature increase, the left SST $\left(6.3 \pm 2.6^{\circ} \mathrm{C}, \mathrm{p}<0.01\right)$ and the right SST $\left(1.1 \pm 1.7^{\circ} \mathrm{C}, \mathrm{p}<0.05\right)$ were significantly increased. Both Finometer systolic blood pressure $(\mathrm{SBP})(6 \pm 7.8 \mathrm{mmHg}, \mathrm{p}<0.01)$ and diastolic blood pressure $(\mathrm{DBP})$ $(5 \pm 6.2 \mathrm{mmHg}, \mathrm{p}<0.01)$ significantly increased. Oscillometric DBP also was significantly increased, but Osillometeric SBP did not show significant changes (Table 2, Figure 2 and Figure 3).

Table 2. Descriptive statistics and paired t-tests results of BP, SST and chamber air temperature

\begin{tabular}{|c|c|c|c|c|c|c|}
\hline & & \multicolumn{5}{|c|}{ Cooling } \\
\hline \multicolumn{2}{|c|}{ Parameters } & Before & After & Difference & $\mathbf{t}$ & $\mathbf{p}$ \\
\hline Finometer & SBP & $122 \pm 14.6$ & $133 \pm 12.4$ & $10 \pm 9.0$ & 4.64 & $* *$ \\
\hline$(\mathrm{mmHg})$ & DBP & $78 \pm 14.0$ & $85 \pm 12.2$ & $7 \pm 6.0$ & 4.61 & $* *$ \\
\hline Oscillometric & SBP & $116 \pm 9.8$ & $116 \pm 9.8$ & $0 \pm 6.9$ & -0.22 & 0.83 \\
\hline$(\mathrm{mmHg})$ & DBP & $74 \pm 8.5$ & $72 \pm 9.7$ & $-2 \pm 5.8$ & -1.12 & 0.28 \\
\hline Temperature & Left SST & $33.3 \pm 1.5$ & $24.5 \pm 2.8$ & $-8.8 \pm 2.5$ & -14.26 & $* *$ \\
\hline \multirow[t]{3}{*}{$\left({ }^{\circ} \mathrm{C}\right)$} & Right SST & $33.3 \pm 1.2$ & $31.7 \pm 1.8$ & $-1.6 \pm 1.4$ & -4.60 & $* *$ \\
\hline & Chamber Temp & $24.2 \pm 0.5$ & $14.8 \pm 0.5$ & $-9.4 \pm 0.7$ & -50.56 & $* *$ \\
\hline & & \multicolumn{5}{|c|}{ Heating } \\
\hline \multicolumn{2}{|c|}{ Parameters } & Before & After & Difference & $\mathbf{t}$ & $\mathbf{p}$ \\
\hline Finometer & SBP & $122 \pm 12.1$ & $128 \pm 12.4$ & $6 \pm 7.8$ & 3.03 & $* *$ \\
\hline$(\mathrm{mmHg})$ & DBP & $77 \pm 12.0$ & $83 \pm 10.3$ & $5 \pm 6.2$ & 3.46 & $* *$ \\
\hline Oscillometric & SBP & $118 \pm 10.7$ & $117 \pm 9.9$ & $-2 \pm 6.0$ & -1.01 & 0.33 \\
\hline$(\mathrm{mmHg})$ & DBP & $76 \pm 14.6$ & $71 \pm 11.6$ & $-5 \pm 7.1$ & -2.89 & $*$ \\
\hline Temperature & Left SST & $32.2 \pm 2.4$ & $38.5 \pm 1.5$ & $6.3 \pm 2.6$ & 9.69 & $* *$ \\
\hline \multirow[t]{2}{*}{$\left({ }^{\circ} \mathrm{C}\right)$} & Right SST & $32.5 \pm 2.1$ & $33.6 \pm 1.1$ & $1.1 \pm 1.7$ & 2.61 & $*$ \\
\hline & Chamber Temp & $24.7 \pm 0.3$ & $44.3 \pm 1.2$ & $19.5 \pm 1.2$ & 63.25 & $* *$ \\
\hline
\end{tabular}

SBP: systolic blood pressure, DBP: diastolic blood pressure, SST: skin surface temperature, Before: 0 min data, After: $20 \mathrm{~min}$ data, Difference $=$ After-Before, ${ }^{*} \mathrm{p}<0.05, * * \mathrm{p}<0.01$. 


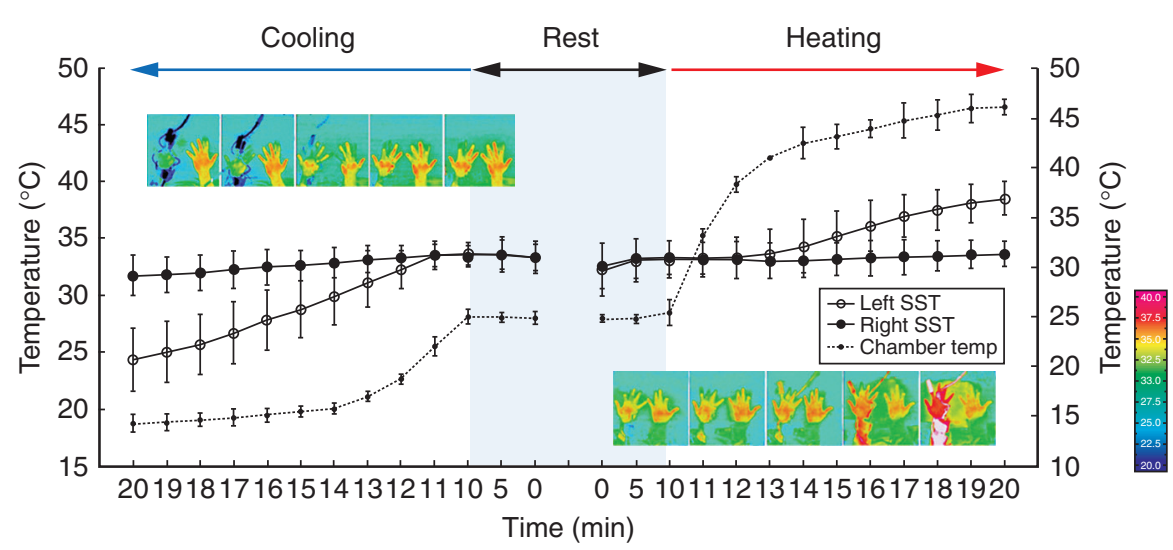

Figure 2. Means and standard deviations of SST and the chamber air temperature, and SST thermograms by infra-red camera.

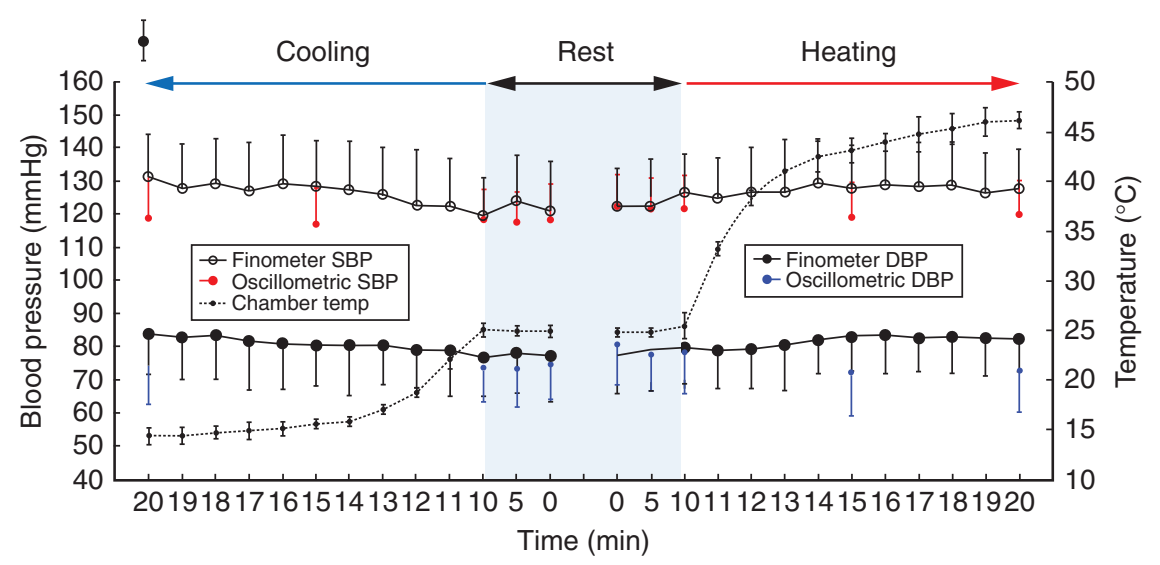

Figure 3. Means and standard deviation of $\mathrm{BP}$ and the chamber air temperature.

\subsection{PPG Components and SST}

PPG components were composed of DC an AC components. DC component includes the foot value and peak value, but AC component had only the gap value. These 3 classified components were found in the left hand. For the initial $10 \mathrm{~min}$ and the rest period, data were individually averaged and set as the reference value of 1 , because the absolute value of the component of each participant was different and could not be compared. Data of the subsequent $10 \mathrm{~min}$ were normalized by the initial value. As shown in Table 3, the ranges of variation in SST and N were different for each participant because of individual physiological responses.

Significant negative correlations $(\mathrm{p}<0.01)$ between DC components and SST were calculated in ranges between -0.634 and -0.980 for DC foot, and between -0.594 and -0.980 for DC peak. All subjects had positive correlations between AC component 
Table 3. Individual correlations between normalized PPG components and SST

\begin{tabular}{|c|c|c|c|c|c|c|c|}
\hline \multirow[b]{2}{*}{ Subject } & \multicolumn{2}{|c|}{ DC foot } & \multicolumn{2}{|c|}{ DC peak } & \multicolumn{2}{|c|}{ AC gap } & \multirow[b]{2}{*}{$\mathbf{N}$} \\
\hline & $\mathbf{r}$ & $\mathbf{p}$ & $\mathbf{r}$ & $\mathbf{p}$ & $\mathbf{r}$ & $\mathbf{p}$ & \\
\hline 1 & -0.947 & & -0.943 & & 0.941 & $* *$ & 15 \\
\hline 2 & -0.701 & & -0.692 & & 0.718 & $* *$ & 16 \\
\hline 3 & -0.937 & & -0.938 & & 0.872 & $* *$ & 12 \\
\hline 4 & -0.810 & & -0.812 & & 0.236 & 0.290 & 19 \\
\hline 5 & -0.711 & & -0.717 & & 0.414 & 0.062 & 12 \\
\hline 6 & -0.634 & & -0.594 & & 0.694 & $* *$ & 12 \\
\hline 7 & -0.768 & & -0.712 & & 0.894 & $* *$ & 9 \\
\hline 8 & -0.939 & $* *$ & -0.945 & $* *$ & 0.720 & $* *$ & 18 \\
\hline 9 & -0.980 & & -0.981 & & 0.936 & $* *$ & 16 \\
\hline 10 & -0.973 & & -0.975 & & 0.315 & 0.190 & 11 \\
\hline 11 & -0.790 & & -0.793 & & 0.689 & $* *$ & 14 \\
\hline 12 & -0.842 & & -0.838 & & 0.817 & $* *$ & 17 \\
\hline 13 & -0.946 & & -0.941 & & 0.924 & $* *$ & 18 \\
\hline 14 & -0.937 & & -0.945 & & 0.747 & $* *$ & 10 \\
\hline 15 & -0.895 & & -0.888 & & 0.914 & $* *$ & 13 \\
\hline 16 & -0.943 & & -0.943 & & 0.108 & 0.624 & 14 \\
\hline
\end{tabular}

r: correlation coefficient, $* * \mathrm{p}<0.01$.

and SST in ranges between 0.108 and 0.941 , but only those of 12 subjects were significant $(\mathrm{p}<0.01)$.

\subsection{Cardiovascular Parameter Changes During Finger SST Change}

Same as PPG components analyses, for the initial $10 \mathrm{~min}$ and the rest period, data were individually averaged, and set as the reference value of 1 , because the absolute value of the component of each participant was different and could not be compared. Data of the subsequent 10 min were normalized by the initial value. As shown in Table 4, the ranges of variation in SST and N were different for each participant because of individual physiological responses.

Significant negative correlations $(\mathrm{p}<0.01)$ were calculated in the range between 0.443 and -0.904 . Correlations between HR and SST, between SV and SST, and between $\mathrm{CO}$ and SST were significant in individuals, but these were not fixed correlations for all subjects.

\section{DISCUSSION}

The initial $10 \mathrm{~min}$ before the heating and cooling periods did not show any significant variation in all parameters. Although some individual vasoconstrictions and vasodilatations appeared, they were caused by the natural process of individual body temperature control. The overall data trend was not affected by these results. 
Table 4. Correlations between Finometer cardiovascular parameters and SST

\begin{tabular}{|c|c|c|c|c|c|c|c|c|c|}
\hline \multirow[b]{2}{*}{ Subject } & \multicolumn{2}{|c|}{ HR } & \multicolumn{2}{|c|}{ SV } & \multicolumn{2}{|c|}{$\mathrm{CO}$} & \multicolumn{2}{|c|}{ TPR } & \multirow[b]{2}{*}{$\mathbf{N}$} \\
\hline & $\mathbf{r}$ & $\mathbf{p}$ & $\mathbf{r}$ & $\mathbf{p}$ & $\mathbf{r}$ & $\mathbf{p}$ & $\mathbf{r}$ & $\mathbf{p}$ & \\
\hline 1 & 0.272 & 0.198 & -0.117 & 0.585 & 0.048 & 0.825 & -0.466 & $*$ & 15 \\
\hline 2 & 0.593 & $* *$ & -0.806 & $* *$ & -0.736 & $* *$ & -0.654 & $* *$ & 16 \\
\hline 3 & -0.213 & 0.396 & -0.099 & 0.696 & -0.172 & 0.495 & -0.504 & $*$ & 12 \\
\hline 4 & -0.637 & $* *$ & -0.521 & $*$ & -0.701 & $* *$ & -0.770 & $* *$ & 19 \\
\hline 5 & 0.752 & $* *$ & 0.110 & 0.636 & 0.645 & $* *$ & -0.904 & $* *$ & 12 \\
\hline 6 & 0.514 & $* *$ & -0.120 & 0.569 & 0.382 & 0.060 & -0.800 & $* *$ & 12 \\
\hline 7 & 0.673 & $* *$ & 0.634 & $* *$ & 0.768 & $* *$ & -0.849 & $* *$ & 9 \\
\hline 8 & 0.368 & 0.084 & 0.288 & 0.183 & 0.427 & $*$ & -0.809 & $* *$ & 18 \\
\hline 9 & -0.070 & 0.755 & 0.597 & $* *$ & 0.408 & 0.060 & -0.629 & $* *$ & 16 \\
\hline 10 & 0.804 & $* *$ & 0.444 & 0.057 & 0.856 & $* *$ & -0.758 & $* *$ & 11 \\
\hline 11 & 0.840 & $* *$ & -0.488 & $*$ & 0.107 & 0.626 & -0.443 & $*$ & 14 \\
\hline 12 & 0.791 & $* *$ & -0.331 & 0.099 & 0.632 & $* *$ & -0.878 & $* *$ & 17 \\
\hline 13 & -0.466 & $*$ & 0.813 & $* *$ & 0.566 & $* *$ & -0.812 & $* *$ & 18 \\
\hline 14 & -0.759 & $* *$ & 0.496 & $*$ & 0.152 & 0.477 & -0.630 & $* *$ & 10 \\
\hline 15 & -0.209 & 0.350 & 0.349 & 0.111 & 0.251 & 0.260 & -0.760 & $* *$ & 13 \\
\hline 16 & 0.256 & 0.239 & -0.769 & $* *$ & -0.384 & 0.071 & -0.754 & $* *$ & 14 \\
\hline
\end{tabular}

HR: heart rate, SV: stroke volume, CO: cardiac output, TPR: total peripheral resistance; r: correlation coefficient; $* \mathrm{p}<0.05, * * \mathrm{p}<0.01$.

SSTs decreased in the cooling process and increased in the heating process along with the chamber air temperature. SST data measured by contact temperature sensors were compared with SST thermogram data using an infrared camera, and this comparison was performed under the assumption that all fingers have similar SST distributions. SST of the left hand that was directly exposed to temperature change in the chamber had faster changes than the right hand outside the chamber. SST thermogram changes were identical to SST sensor changes. SST gradually changed after a sudden initial change because of the physiological thermo-natural process of the limbs. However, reduction ratios in each individual were not identical due to individual physiological differences [16]. A previous study [17] demonstrated that the data of noncooled right hand did not significantly change, but could demonstrate a similar decreasing trend. A continuous increasing trend also could be found in the heating process. Although the cooling period did not affect the right hand directly, the nonexperimental hand was affected by the mechanism of body temperature control, so correlated results were produced.

In the initial $10 \mathrm{~min}$, similar values of BP were obtained in both the oscillometric and Finometer data; however, the values were significantly different when the chamber air temperature was changed. The oscillometric BP was not affected but Finometer BP, which employed PPG estimation method, was affected by changes in SST because changes in blood flow and blood vessels were generated by the temperature adaptation mechanism. 
changes in blood flow and blood vessels were generated by the temperature adaptation mechanism.

In the temperature range between $22^{\circ} \mathrm{C}$ and $40^{\circ} \mathrm{C}$ of the left hand, PPG DC components were significantly decreased but PPG AC component was generally increased. It is well known that cooling of the blood vessel walls causes the loss of contractive capacity, while heating increases its capacity. The results of the present experiments demonstrated good agreement with the above phenomenon for the cooling process, but weak agreement for the heating process. This supports the phenomenon of stronger protection against cold than heat [18]. The results also reflected that the PPG component changes were linked to changes in the body temperature and the theory that maintenance of body temperature under the increase and decrease of heat loss was mainly achieved through the blood flow control [16].

Four Finometer cardiovascular parameters show only changes in the entire body but not local changes [19]. The overall changes in HR, SV, and CO did not show significant correlations with SST, while TPR showed an increasing trend. This result demonstrated that local contraction of blood vessels due to local changes in SST caused changes in the total cardiovascular resistance because the hands have functional importance and excellent control of heat loss $[16,18]$.

Limitations of this pilot study include the small number of subjects and not considering the gender difference. The present results should be further confirmed in future studies taking into account these limitations.

\section{CONCLUSION}

This study investigated effects of changing skin surface temperature on BP, PPG and cardiovascular parameters such as heart rate (HR), stroke volume (SV), CO and total peripheral resistance (TPR). The clinical physiological changes were explained by changes in cardiovascular status in terms of changes in time, chamber air temperature, and SST of both hands. Changes in cardiovascular characteristics and peripheral vessels by SST change were demonstrated. It is concluded that temperature should be taken into account in evaluating the status and functioning of the cardiovascular system using PPG components. Temperature is an important factor for more accurate evaluation of the cardiovascular system.

\section{ACKNOWLEDGEMENTS}

This work was partly supported by the Bio-Meditech Regional Innovation Center at Eulji University, under the Regional Innovation Center Program (2013-02-01) of the Ministry Of Trade, Industry and Energy, R. O. Korea.

\section{CONFLICT OF INTEREST}

The authors indicated no potential conflicts of interest.

\section{REFERENCES}

[1] Anderson RR, Parrish JA. The optics of human skin. J Invest Dermatol, 1981, 77:13-9.

[2] Spigulis J. Optical noninvasive monitoring of skin blood pulsations. Appl Optics, 2005, 44:1850-7. 
[3] Davis DL, Baker CN. Comparison of changes in blood volume and opacity in dog digital pad and tongue. J Appl Physiol, 1969, 27:613-8.

[4] Zhang Q, Lindberg G, Kadefors R, Styf J. A non-invasive measure of changes in blood flow in the human anterior tibial muscle. Eur J Appl Physiol, 2001, 84:448-52.

[5] Kraitl J, Ewald H, Gehring H. An optical device to measure blood components by a photoplethysmographic method. J Opt A Pure Appl Opt, 2005, 7:S318-S24.

[6] Teng XF, Zhang YT. The effect of contacting force on photoplethysmographic signals. Physiol Meas, 2004, 25:1323-35.

[7] Nitzan M, Babchenko A, Khanokh B. Very low frequency variability in arterial blood pressure and blood volume pulse. Med Biol Eng Comput, 1999, 37:54-8.

[8] Maeda Y, Sekine M, Tamura T. Relationship between measurement site and motion artifacts in wearable reflected photoplethysmography. J Med Syst, 2010, 34.

[9] Incheol J, Sukhwan J, Daeja Um, Joonghwan Oh, Hyungro Y. Non-Invasive estimation of systolic blood pressure and diastolic blood pressure using photoplethysmograph components. Yonsei Med J, 2010, 51(3):345-53.

[10] Grindheim G, Estensen ME, Langesaeter E, Rosseland LA, Toska K. Changes in blood pressure during healthy pregnancy: a longitudinal cohort study. J Hypertens, 2012 Feb, 30(2)

[11] Lukácsovits J, Carlucci A, Hill N, Ceriana P, Pisani L, Schreiber A, Pierucci P, Losonczy G, Nava S. Physiological changes during low- and high-intensity noninvasive ventilation. Eur Respir J, 2012 Apr, 39(4):869-75.

[12] Greaney JL, Matthews EL, Boggs ME, Edwards DG, Duncan RL, Farquhar WB. Exaggerated exercise pressor reflex in adults with moderately elevated systolic blood pressure: role of purinergic receptors. Am J Physiol Heart Circ Physiol, 2014 Jan, 306(1)

[13] Fukushima H, Kawanaka H, Bhuiyan MS, Oguri K. Cuffless blood pressure estimation using only photoplethysmography based on cardiovascular parameters. Conf Proc IEEE Eng Med Biol Soc, 2013 , 2013:2132-5

[14] Elvan-Taspnar A, Uiterkamp L, Sikkema JM, Bots ML, Koomans HA, Bruinse HW et al. Validation and use of the FinometerTM for blood pressure measurement in normal, hypertensive and preeclamptic pregnancy. J Hypertens, 2003, 21:2053-60.

[15] Jason WD, Paul AM, James DS, Charles LS. Effects of caffeine on blood pressure, heart rate, and forearm blood flow during dynamic leg exercise. J ApplPhysiol, 1998, 85:154-9.

[16] Huizenga C. A model of human physiology and comfort for assessing complex thermal environments. Build Environ, 2001, 36:691-9.

[17] Stroud MA. Digital and brachial artery blood pressure measurements during peripheral, cold-induced vasoconstriction. Eur J ApplPhysiol, 1994, 68:134-38.

[18] Huizenga C. Skin and core temperature response to partial- and whole-body heating and cooling. J ThermBiol, 2004, 29:549-58.

[19] Parati G, Ongaro G, Bilo G, Glavina F, Castiglioni P, Di Rienzo M et al. Non-invasive beat-to-beat blood pressure monitoring: new developments. Blood Press Monit, 2003, 8:31-6. 


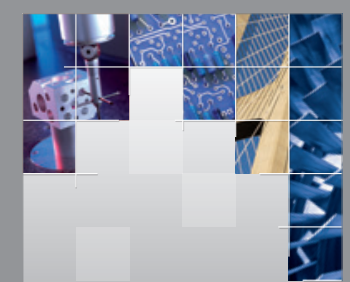

\section{Enfincering}
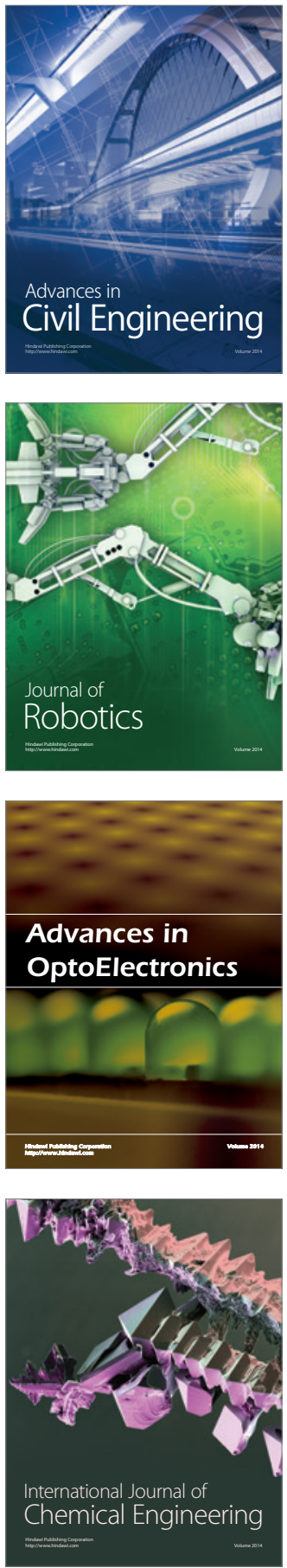

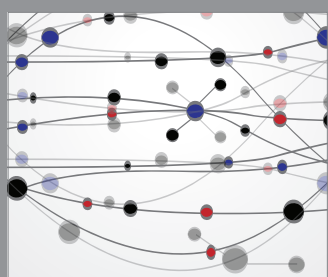

The Scientific World Journal

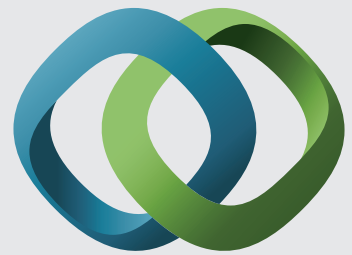

\section{Hindawi}

Submit your manuscripts at

http://www.hindawi.com
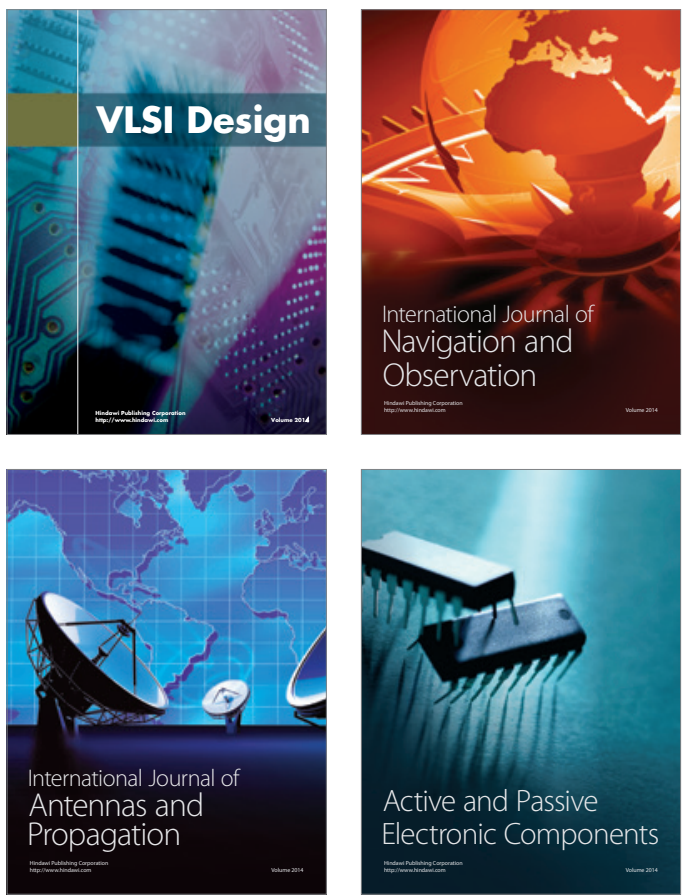
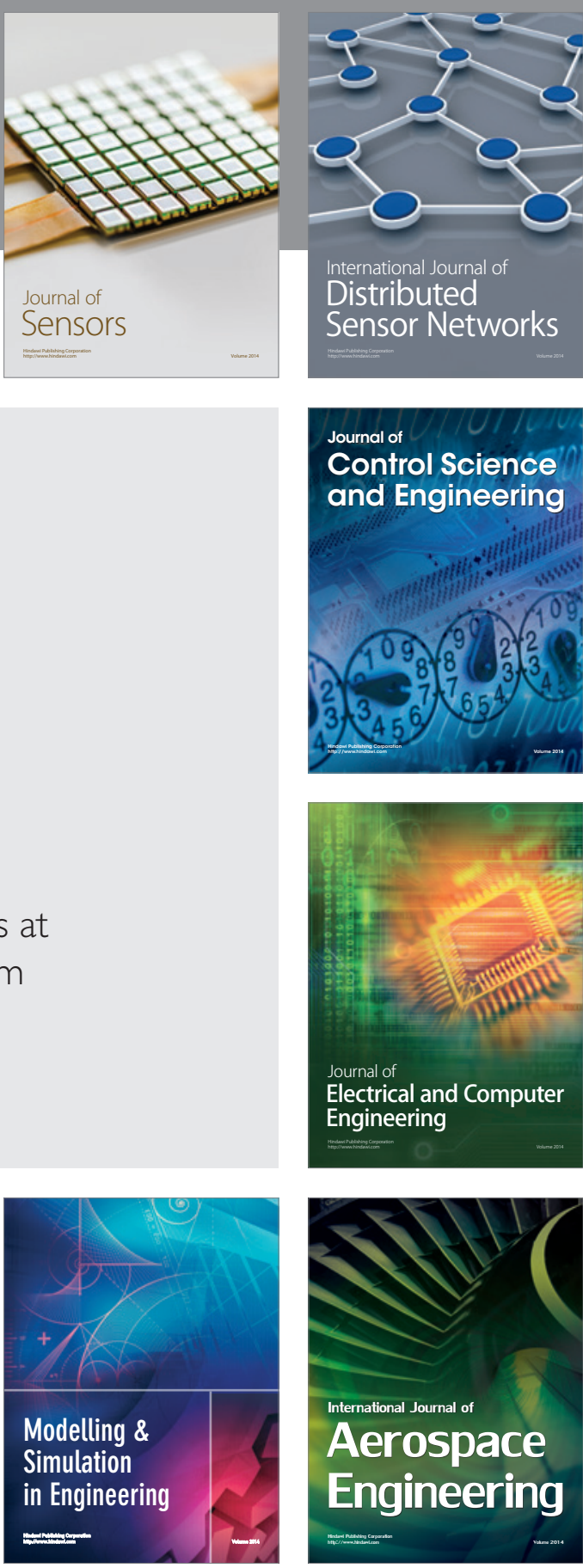

International Journal of

Distributed

Sensor Networks

Journal of

Control Science

and Engineering
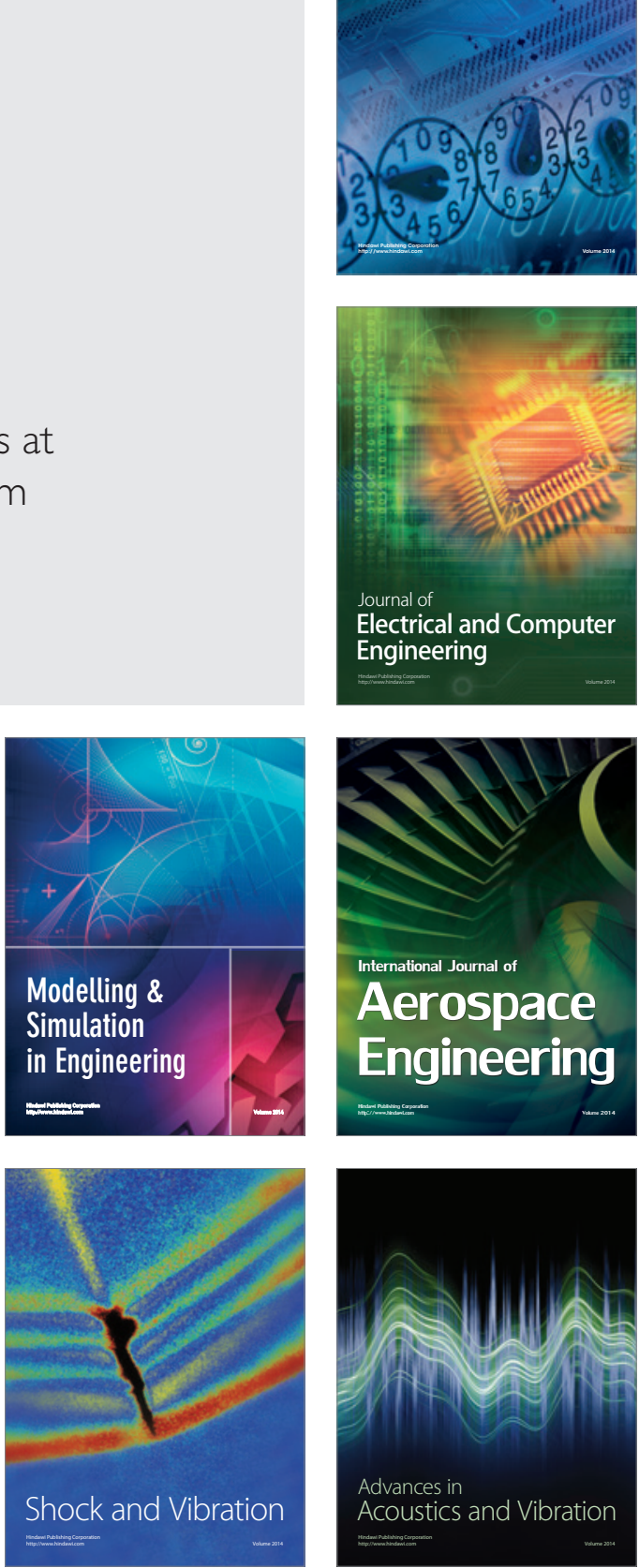